\title{
IOT BASED WEED DETECTION USING IMAGE PROCESSING AND CNN
}

\author{
Shweta Kulkarni \\ Dept. of Computer Science and Engineering \\ Center Of Post Graduate Studies \\ VTU Belagavi
}

\begin{abstract}
Farming is the source of income for more than half of the Indian population. One of the serious issues in agriculture is the control of weeds growing among the plantation crops. At present weeds are being removed manually by farmers wherever possible, or weed killers/herbicides are being sprayed all over the field to keep them under control. This technique is very inefficient because chemicals are being sprayed on plantation crops also, which leads to, polluting the environment and health problems in humans. To avoid these consequences, a smart weed control system should be deployed. This paper focuses on detecting the weeds in the crop using convolutional neural network ,Image processing and IOT. CNN model is first trained by giving large images of weed and crop. This trained CNN model is deployed onto Raspberry pi. Images from camera is sent to raspberry pi based Machine learning system. Raspberry-pi performs Image segmentation, by dividing the image into small segments. The Segmentation Algorithm used is Watershed Segmentation Algorithm. Each segment is passed onto Trained CNN model for classifying as weed or crop. If it is weed, the area is marked in the original image as weed. In this manner all the weed segments are marked and the marked image can be sent to farmers through Email. The system was trained using 250 images of weed and crop and has given an Average Accuracy of $85 \%$,Average False ratio of $7 \%$,Average False Acceptance ratio of $2.6 \%$.
\end{abstract}

Keywords-Convolutional Neural Network, watershed segmentation Algorithm, Raspberry Pi

\section{INTRODUCTION}

Farming is the foundation of Indian economy. It is a source of income for more than half of the Indian population. One of the serious issues in agribusiness is the control of weeds growing among the plantation crops. A weed is a plant which bothers many farmers. "a plant in the wrong spot". Numerous plants that people broadly see as weeds are deliberately developed in farms, in which case they are called useful weeds. The term weed additionally is connected to any plant that develops or replicates forcefully. Weeds also require all essential nutrients that are required by plantation crops. Weeds if not checked may compete with other plantation crops for nutrients, space, water ,soil, and restrict their growth. Weeds play as host for plant pathogens which infect and degrade the quality of plantation crops. some weeds may also cause root damage to drainage pipes, road surfaces etc.

\author{
Dr. S.A Angadi \\ HOD, Computer Science and Engineering \\ Director (I/C) ITISMU \\ VTU Belagavi
}

\begin{abstract}
At present weeds are being removed manually by farmers wherever possible, or weed killers/herbicides are being sprayed all over the field to keep them under control This technique is very inefficient because chemicals are being sprayed on plantation crops also, which leads to, polluting the environment and health problems in humans . To avoid these consequences, a smart weed control system should be deployed. The system must be capable of locating weeds in the field and alerting the farmers about exact locations of these weeds. So that herbicides are sprayed only in those locations. It focuses on reducing use of pesticides that harms the normal growth of plants. as well as serious health issues posed in humans.
\end{abstract}

This paper focuses on detecting the weeds in the crop using convolutional neural network ,Image processing and IOT. Image acquisition is accomplished by the Camera attached to Raspberry pi based Machine learning module. Convolutional Neural Network is used for weed detection using image processing. CNN model is first trained by giving large images of weed and crop. This CNN model is now deployed onto raspberry pi. Images from camera is sent to raspberry pi at regular intervals.Raspberry-pi performs Image segmentation, by dividing the image into small segments. The Segmentation Algorithm used is Watershed Segmentation Algorithm. Each segment is passed onto Trained CNN model for classifying as weed or crop. If it is weed, the area is marked in the original image as weed. In this manner all the weed segments are marked and the marked image is sent to farmers through Email. Section I includes the Introduction. Section II focuses on the Literature Survey. Section III highlights the Proposed Methodology while Section IV presents the Experimentation and Results and Section V concludes the paper.

\section{RELATED WORK}

Many deep learning based approaches have been proposed for weed detection and classification. Below Mentioned are some of the papers which I have made a survey .

Kamarul Hawari Ghazali et. al. [1] proposed work on machine vision exactness for computerized weed control framework. In this work, a keen ongoing framework is proposed for weeding system in oil palm plantation. utilizing picture handling has been created to distinguish and separate the weed sorts to be specific as thin and expansive. 


\section{International Journal of Engineering Applied Sciences and Technology, 2019 Vol. 4, Issue 3, ISSN No. 2455-2143, Pages 606-609 \\ Published Online July 2019 in IJEAST (http://www.ijeast.com)}

Amir H. Kargar B, Ali M. Shirzadifar [2] proposed a work on Automatic Weed Detection System And Smart Herbicide Sprayer Robot For Corn Fields. This paper centers around building up a automatic plantation system. Here products of the soil in the estate is recognized and herbicide is showered for the weed influenced area.

Muhammad H. Siddiqi et. al. [3] has proposed a methodology on Weed Recognition Based on Erosion and Dilation Segmentation Algorithm. The reason for this paper is to build up a calculation for constant that isn't an independent robot however to explore a continuous machine vision framework. The framework contains a CCD camera mounted before the tractor at an edge of 45 degree at a separation of 4 meter long from the earth, which is utilized for the arrangement of two sorts of weeds (expansive and restricted) by utilizing the proposed picture preparing strategy Erosion pursued by Dilation.

Asnor Juraiza Ishak et. al. [4] proposed work on Weed Detection using Quadratic Polynomial and ROI Techniques. this paper considers a bend identification strategy dependent on the quadratic polynomial procedure which incorporate the utilization of the locale of-interests (ROI) method. The ROI method makes picture subsets by choosing regions of the showed picture. The ROIs are regularly used to separate measurements for picture tasks, for example, order. All things considered, This paper is to introduce a novel use of bend identification highlight extraction strategy in weed order.

Xavier P. Burgos-Artizzu et. al. [5] proposed work on Realtime picture handling for yield/weed separation in maize fields. The framework comprises of two free subsystems, a quick picture handling conveying results continuously (Fast Image Processing, FIP), and a slower and progressively precise preparing (Robust Crop Row Detection, RCRD) that is utilized to address the primary subsystem's mix-ups. This mix creates a framework that accomplishes generally excellent outcomes under a wide assortment of conditions.

\section{PROPOSED METHODOLOGY}

The proposed system design is shown in Fig 1.

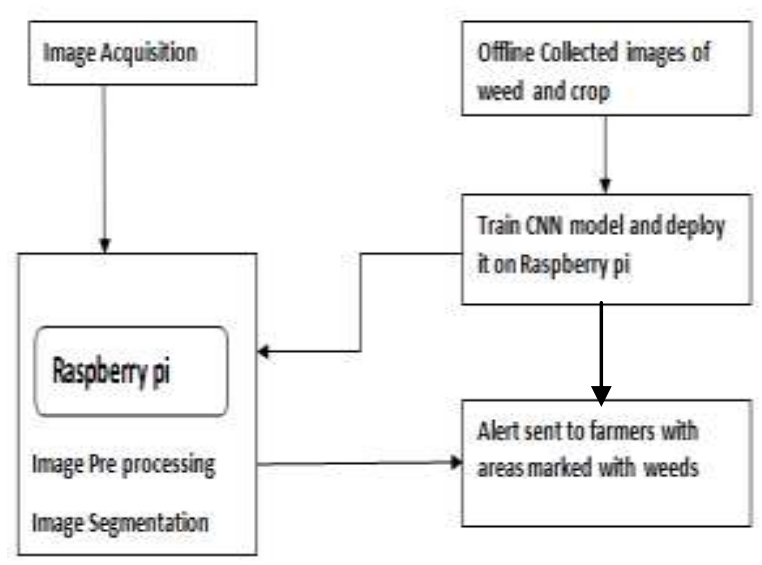

Fig 1: Proposed System Design

The Proposed system design consists of Raspberry pi and camera .Image acquisition is done using camera. The image captured by camera is sent to raspberry pi which performs Image preprocessing and Image segmentation The proposed methodology consists of a set of stages starting from collecting crop and weed images. The main steps are shown below.

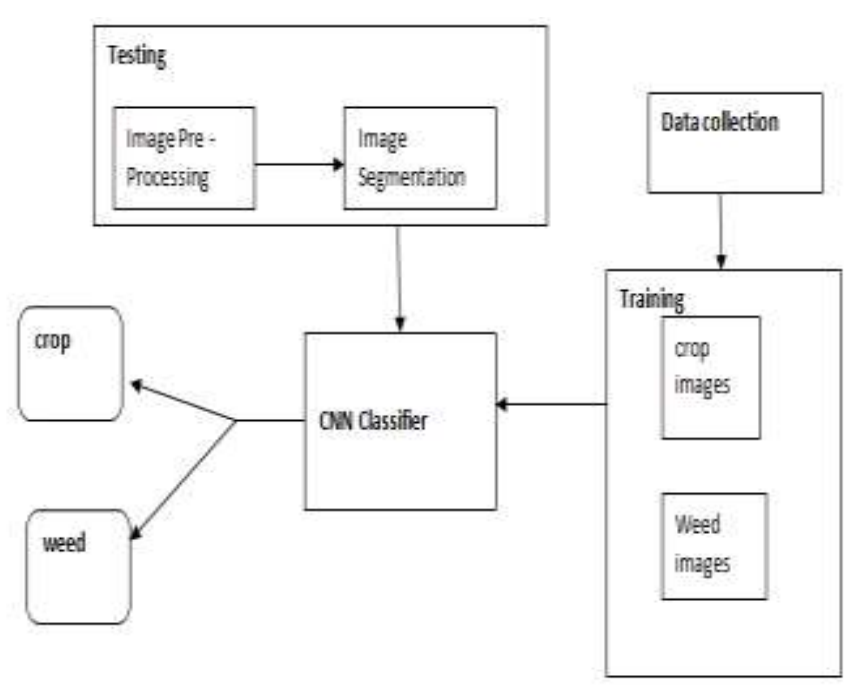

Fig 2:Block Schematic Diagram

\section{A. Collection of weed and crop images:}

Datasets consisting of weed and crop images are required for training Convolutional neural Network. Images will grouped into 2 classes. Crop images and weed images are stored in separate folder and are given for training CNN.

\section{B. Training Convolutional Neural Network:}

(i) Convolutional neural network

Convolutional neural network is special architecture used for deep learning. It is used to detect objects. CNN learns to identify objects without manual assistance. A convolutional neural system can have many layers that each figure out how to distinguish various features of an image. Filters are applied to each training image at different resolutions, and the output of each convolved image is used as the input to the next layer. The starting layers can detect very simple features, such as brightness and edges, and as the layers increase more complex features that uniquely define the object can be detected. The segmented image will be processed with CNN using ReLU and pooling layers.

(ii) Prepare Training and Test Image Sets

Split the images into training and validation datasets. Pick $70 \%$ of images from each crop and weed image dataset set for the training and the rest $30 \%$, for the validation data. $\mathrm{CNN}$ is trained using the training and validation sets. 


\section{Deploy Trained CNN network onto Raspberry pi:}

Once the CNN network is trained it has to be deployed on Raspberry pi. Training the CNN network on raspberry pi takes huge time since RAM is very less. Hence CNN network is trained on laptop and then the .h5 file which contains weights of the neural network is deployed on raspberry pi.

\section{Image Segmentation:}

Image segmentation is one of the most important part of image processing. The purpose of image segmentation is to divide an original image into equal homogeneous regions. There exist several different approaches for image segmentation methods .Watershed Image segmentation Algorithm is studied in this paper. watershed algorithm can be implemented by applying flooding process on grey tone image. This flooding procedure can be performed by utilizing essential morphological activities. The total change consolidates a pre-processing and post-processing stage that manages implanted issues, for example, edge ambiguity,etc Watershed Transform can be connected to gray scale pictures, textural pictures and binary pictures. watershed algorithm marks each of the segments with unique labels. The marked segments are stored in .csv file the CNN network reads each of the segments from the csv file and classifies it either as crop or weed.

\section{E. Sending Alerts to farmers:}

CNN network classifies each segment as either crop or weed. If it's a weed the segment is marked in the original image as weed. The marked image with weed is sent as Email to farmers through Raspberry pi GSM module.

\section{Performance of the proposed system:}

The Performance of the proposed method is described below

The accuracy of the proposed system can be calculated as follows.

Accuracy $=$ Number of weed segments correctly identified / Total number of weed segments.

The False Rejection Ratio (FRR) of the proposed system can be calculated as follows.

FRR $=$ Number of weed segments identified as non weed / Total number of weed segments.

The False Acceptance Ratio (FAR) of the proposed system can be calculated as follows.

FAR $=$ Number of non weed segments identified as weed / Total number of segments.

\section{IV.EXPERIMENTAL RESULTS}

The proposed system uses CNN for identifying weeds in agriculture farms. We have used 250 sample images for training CNN network and experiments were conducted on 20 images which in turn produced an average accuracy of
$85 \%$. The following figures will focus more on weed detection in crops.

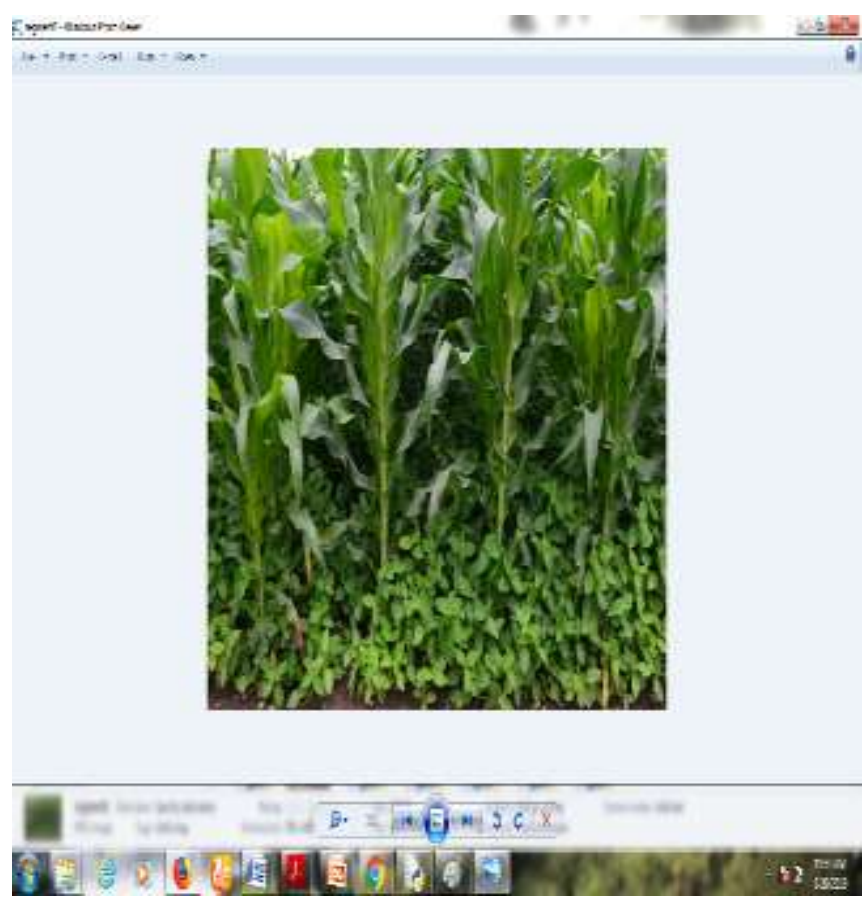

Fig 3: The image with maize crop surrounded by weed

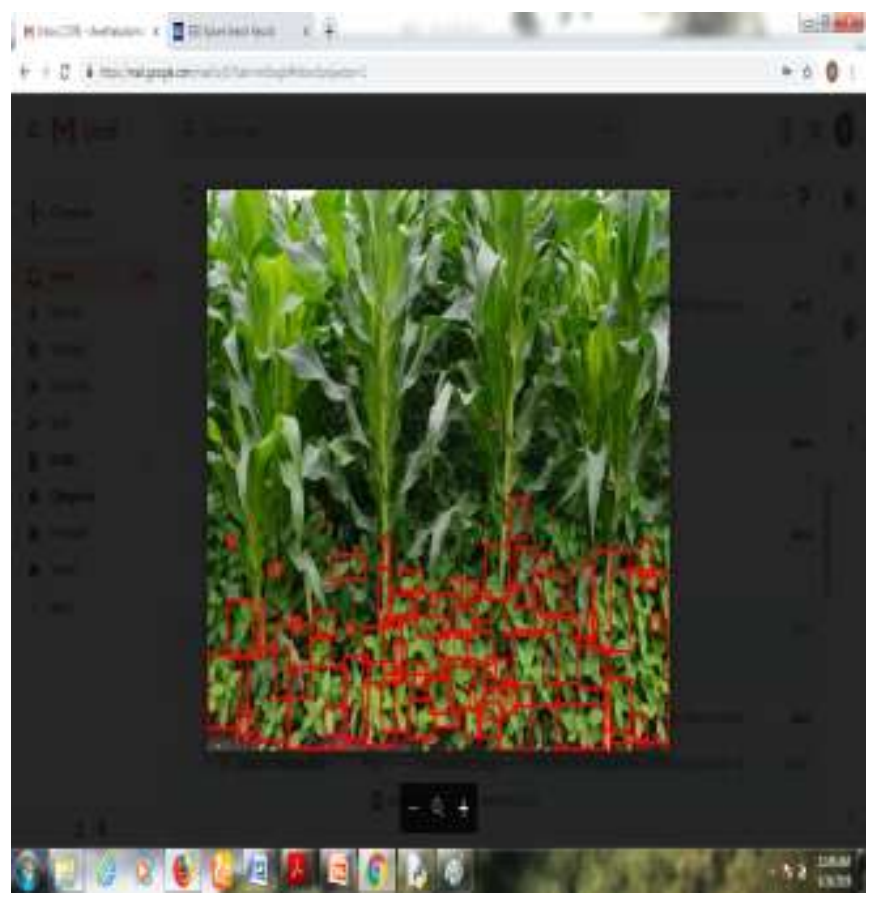

Fig 4: The image with maize crop with detected weed

The following table gives different images along with actual weed segments, predicted weed segments and the Accuracy of prediction is given. 


\section{International Journal of Engineering Applied Sciences and Technology, 2019 Vol. 4, Issue 3, ISSN No. 2455-2143, Pages 606-609 \\ Published Online July 2019 in IJEAST (http://www.ijeast.com)}

\begin{tabular}{|c|c|c|c|}
\hline Image & $\begin{array}{c}\text { No of reed segments } \\
\text { actually present }\end{array}$ & $\begin{array}{c}\text { No. of Weed } \\
\text { segments predicted }\end{array}$ & Accuracy \\
\hline Image 1 & 42 & 39 & $92 \%$ \\
\hline Image 2 & 34 & 31 & $91 \%$ \\
\hline Image 3 & 36 & 35 & $97 \%$ \\
\hline Image 4 & 47 & 4 & $93 \%$ \\
\hline Image5 & 59 & 55 & $93 \%$ \\
\hline Image6 & 61 & 56 & $91 \%$ \\
\hline Image 7 & 44 & 40 & $90 \%$ \\
\hline Image 8 & 21 & 20 & $95 \%$ \\
\hline Image 9 & 34 & 32 & $94 \%$ \\
\hline Image 10 & 17 & 16 & $94 \%$ \\
\hline
\end{tabular}

Table 6.1 : Weed Detection Analysis

The Average False rejection ratio of the proposed system is $7 \%$

The Average False Acceptance ration of the proposed system is $2.6 \%$

\section{CONCLUSION}

The proposed system, IOT Based Weed detection using CNN and Image processing uses Convolutional Neural network for detecting weeds. Farmers can sit at home and can monitor the growth of crops and weeds. It is not necessary for farmers to visit the farms everyday to check the growth of weeds. Framework can be extended to give information to Robot and drones to spray herbicides in only weed detected areas and a Mobile Application can be developed where our Proposed framework can send Weeds detected areas to mobile App.

Weed control and detection has become one of the most interesting domains. Preventing the use of chemicals as well as saving money are some reasons of this trend. Through this project it can be seen that Internet of Things and Image Processing along with CNN can be combined and implanted in the field of weed control to get good results. Farmers are connected and aware of the conditions of the agricultural field at anytime and anywhere in the world.

\section{ACKNOWLEDGEMENT}

I would like to express my special thanks of gratitude to my guide and HOD Dr S.A.Angadi, who gave me the opportunity to do this wonderful project on the topic IOT Based Weed Detection using Image Processing and CNN which also helped me in doing lots of research and I came to know many things I am really thankful to them and even my friends.

\section{REFERENCES}

[1] Kamarul Hawari Ghazali, Mohd. Marzuki Mustafa, Aini Hussain (2008) "Machine Vision System for Automatic Weeding Strategy in Oil Palm Plantation using Image Filtering Technique" American-Eurasian J. of Agriculture \& Environmental Science, vol. 3, no. 3,

[2] Amir H. Kargar B, Ali M. Shirzadifar, (Feb. 2013) "Automatic Weed Detection System and Smart Herbicide Sprayer Robot for corn fields," IEEE Int. Conf. on Robotics and Mechatronics, Tehran, pp. $468-473$.

[3] Muhammad H. Siddiqi, Irshad Ahmad, Suziah Bt Sulaiman (Apr. 2009) "Weed Recognition Based on Erosion and Dilation Segmentation Algorithm," Int. Conf. on Education Technology and Computer, Singapore,

[4]Asnor Juraiza Ishak, Siti Salasiah Mokri, Mohd. Marzuki Mustafa, and Aini Hussain, (Dec. 2007) "Weed Detection utilizing Quadratic Polynomial and ROI Techniques", in Proc. of 5th Student Conf. on Research and Development, Malaysia,

[5] Xavier P. Burgos-Artizzu, Angela Ribeiro, Maria Guijarro, Gonzalo Pajares; (2010 ) "Real- time image processing for crop/weed discrimination in maize fields"; Elsevier;

[6] Ajit G Deshmukh, V.A. Kulkarni, (2013) "Advanced Robotic Weeding System," Trans. on Electrical and Electronics Engineering, vol. 1, no. 3,.

[7] Chengliang Liu, Mingjun Wang, and Jun Zhou,( 2008) "Co-Ordinating control for an agricultural vehicle with individual wheel speeds and steering angles," IEEE Control Systems Magazine,

[8] H. Pota, R. Eaton, J. Katupitiya, S.D. Pathirana, ( Aug. 2007) "Agricultural robotics: A streamlined approach to realization of autonomous farming," 2 nd Int. Conf. on Industrial and Information System, IEEE

[9] A. J. Irías Tejeda, R. Castro Castro, (March 2019)" Algorithm of Weed Detection in Crops by Computational Vision" International Conference on Electronics, Communications and Computers ,IEEE

[10] S Umamaheswari, R Arjun, D Meganathan ( October 2018) " Weed Detection in Farm Crops using Parallel Image Processing" International Conference on Information and Communication Technology (CICT)

[11] Juan Sandino, Felipe Gonzalez ,(2018)" A Novel Approach for Invasive Weeds and Vegetation Surveys Using UAS and Artificial Intelligence" 23rd International Conference on Methods \& Models in Automation \& Robotics (MMAR)

[12] Maged Wafy,Hashem Ibrahim, Enas kamel (2013)" Identification of weed seeds species in mixed sample with wheat grains using SIFT algorithm" 9th International Computer Engineering Conference(ICENCO)

[13] M. Dian Bah ; Adel Hafiane ; Raphael Canals (2017) " Weeds detection in UAV imagery using SLIC and the hough transform" Seventh International Conference on Image Processing Theory, Tools and Applications (IPTA). 